\title{
Approach to multiple sclerosis associated uveitis: 3 case reports
}

\begin{abstract}
Background: Uveitis which is known as inflammation in adjacent ocular structures can be one of the ocular manifestations of multiple sclerosis. It is shown that the prevalence of uveitis in patients suffering from MS is more than the normal population. This case report encompasses the association between the two diseases. The diagnostic and approaching the patients to both disorders are fully discussed.
\end{abstract}

Case presentation: 3 patients with definite MS and uveitis are reported. The first and second patients have the feature of intermediate uveitis and the third one has sclerouveitis. Each patient has a negative laboratory test for inflammatory and infectious diseases.

Conclusion: Uveitis can be considered as an attack of MS, therefore this eye impairment should be managed by an ophthalmologist and then refer back to the neurologist for further MS treatment and management.

Keywords: Uveitis and multiple sclerosis association, demyelination, inflammation, disease management

Abbreviations: MS: Multiple Sclerosis; SUN: Standardization of Uveitis Nomenclature; IRBP: Interphotoreceptore Retinoid-Binding Protein; CXR: Chest X-Ray; IFN: Interferons; CNS: Central Nervous System; DTR: Deep Tendon Reflex; VEPs: Visual Evoked Potentials; OCB: Oligo Clonal Bands; ANA: Anti-Nuclear Antibodies; APS: Antiphospholipid; HTLV1, 2: Human T-cell Leukemia Virus type 1,2; HIV: Human Immunodeficiency Virus; CMV: Cytomegalovirus; DMT: Disease Modifying Therapy

\section{Background}

Multiple Sclerosis (MS) as a chronic demyelinating disorder of the central nervous system (CNS) causes some impairments in vision and ocular system, such as optic neuritis, internuclear ophthalmoplegia, many types of debilitating nystagmus and, less frequently, ocular inflammatory diseases [1]. Windmuller [2] for the first time published a case report of a patient who suffered both choroiditis and MS and therefore, the connection between uveitis and MS has been revealed. Meanwhile, it is noted that by the middle of the $20^{\text {th }}$ century, some types of uveitis, including anterior, intermediate, posterior, and panuveitis were reported in patients having MS [3,4]. The association between MS and uveitis has been reported in different publications. For example, Tola et al [5] studied 110 patients who suffered from MS and estimated that the prevalence of uveitis was nearly 36 percentages $(n=40)$. In the other study which was conducted by Shugaiv et al [6], the prevalence was estimated for about 0.7 percentages ( 41 patients with uveitis in 6024 MS patients). As it was reviewed by Olsen TG et al [7], based on the largest retrospective studies with cohorts of more than 1000 patients, the prevalence of uveitis among MS patients was around $1 \%$ and the prevalence of MS among patients with uveitis is about $1 \%$ as well. On the contrary, in some studies, no possible correlation between the two diseases has been reported [8].

Both diseases are considered as severe disorders; hence, it is crucial to diagnose them at an early stage in order to begin treatment to secure better long term clinical outcomes. As a matter of fact, the term uveitis is also often used to describe inflammation in adjacent ocular structures (the vitreous, the retina and its vessels) which can be presented by various symptoms, such as, blurred vision, pain, photophobia, lacrimation, and congestion [9]. Today, the

\section{Saeed Shahbeigi ${ }^{{ }^{*}}$, Soheila Kianpour Rad', Hosein Pakdaman ${ }^{3}$, Mohammad Hafez Noroozizadeh 4 , Surena Nazarbaghi ${ }^{5}$, Sana Karamolahi ${ }^{6}$, and Ayse Altintas ${ }^{7}$}

${ }^{1}$ Neurologist and Neuroimmunologist, Shahid Beheshti Medical University Research Center, Tehran, Iran ${ }^{2}$ Qazvin University of Medical Science, Qazvin, Iran

${ }^{3}$ Shahid Beheshti Medical University Research Center, Tehran, Iran

${ }^{4}$ Ophthalmologist, Cornea subspecialist, NEGAH Eye Hospital, Tehran, Iran ${ }^{5}$ URMIA University of Medical Sciences, Iran

${ }^{6}$ Azad University of Medical Sciences, Tehran, Iran

${ }^{7} \mathrm{Koc}$ University, School of Medicine, Neurology Department, Istanbul, Turkey

${ }^{*}$ Author for correspondence:

s_shahbeigi@yahoo.com 
Standardization of Uveitis Nomenclature criteria (SUN-criteria) [10] are used for classification of uveitis based on anatomical location, etiology, and cause. The classifications of anatomical location are as follows: 1 . anterior uveitis, including iritis, iridocyclitis and anterior cyclitis, 2. intermediate uveitis, such as, vitritis, posterior cyclitis, pars planitis, hyalitis, 3 . posterior uveitis covers, choroiditis, retinitis, chorioretinitis, retinochoroiditis, neuroretinitis, retinal vasculitis/periphlebitis, and 4. panuveitis which are anterior, intermediate, and posterior inflammation. In addition, in the etiological classification, uveitis is sorted as infectious, non-infectious, and masquerade which could be neoplastic/non-neoplastic [10,11]. Different subtypes of uveitis, which are closely related pathogenetically, are known to be provoked by an autoimmune response against retinal antigens, such as S-antigen, Interphotoreceptor Retinoid-Binding Protein (IRBP), rhodopsin, and recoverin [12]. An in vivo study which was conducted on autoimmune encephalomyelitis (EAE), showed that there were several candidate autoantigens were identified as potential targets of activated autoreactive T-cells in patients with MS. In fact, CNS-inflammation could be accompanied by a vast panuveitis and retinitis, a lesional distribution which cannot be observed in EAE models mediated by myelin-specific T-cells [13]. It is revealed that antigens which are co-expressed in both CNS and retinouvea might be pathogenetically germane to a subgroup of MS patients $[13,14]$. It is suggested that either nerve or eye tissue develop from the same embryonic cells; thus MS and uveitis could be etiologically associated [15]. Although there are several studies reported that MS and uveitis can be considered as coexisting diseases, more evaluations are required for better understanding the exact association. This study aims to evaluate the prevalence of uveitis in MS patients as well as analyzing the recommended approaches in the literature for such co-existing diseases.

\section{Case Presentation}

\section{Case 1}

A 39 years old man with the history of paraparesis and para-paresthesia in 2016, presented a bilateral blurred vision, photophobia, and redness symptoms in 2018. As soon as the uveitis was diagnosed by an ophthalmologist, prednisolone and mycophenolate mofetil was prescribed and referred for further neurological evaluations. The examinations were completely normal, except that bilateral blurry vision and bilateral 3+knee deep tendon reflex (DTR) were observed. Besides, few periventricular and subcortical lesions with no enhancement were seen on the brain MRI, and also on the cervical MRI, few high-signal foci were located without any enhanced lesion (FIGURE 1). The bilateral abnormal Visual Evoked Potential (VEPs) and 5 positive oligoclonal bands (OCB) in CSF were reported. The immunologic tests, including, Antinuclear Antibodies (ANA), Anti-DNA, Antiphospholipid (APS), Anti-Ro (SSA) and Anti-La (SSB), lupus anticoagulant and also the laboratory tests for Human T-cell leukemia virus type 1,2 (HTLV1, 2), Lyme disease, syphilis, toxoplasmosis, human immune deficiency virus (HIV), B and C hepatitis, cytomegalovirus (CMV), tuberculosis were negative.

\section{- Case 2}

A 31 years old lady, who has been diagnosed as MS since 2003, presented by blurred vision and gait impairments. She was given methylprednisolone and became symptoms free at that time and the Disease Modifying Therapy (DMT) was started for her. After 4 months, she developed ocular problems, including, redness, blurred vision and also a pain. The uveitis was diagnosed by an ophthalmologist and the treatment by Avonex was stopped, and then prednisolone and azathioprine have been initiated for 10 years. In 2017, she experienced the second attack as the left hemi-paresthesia which accompanied by gait problem. She had the left hemiparesis and the ataxia 2 months later; therefore, she was given the pulse therapy for $3^{\text {rd }}$ times. She currently has multiple high signal lesions in her either brain or cervical MRI as well as bilateral abnormal VEPs (FIGURE 1). The Immunological and other laboratory tests are normal. Her medication was changed to rituximab due to the progression course of the disease.

\section{- Case 3}

A 27 years old girl with no previous medical history presented left optic neuritis in 2017. In the brain MRI, few high signal foci were seen in the periventricular and the centrum semiovalis and at least two enhanced perpendicular lesions as well but it was noticed that the cervical MRI was normal (FIGURE 1). She was administrated 
FIGURE 1: Brain MRI of the patients.

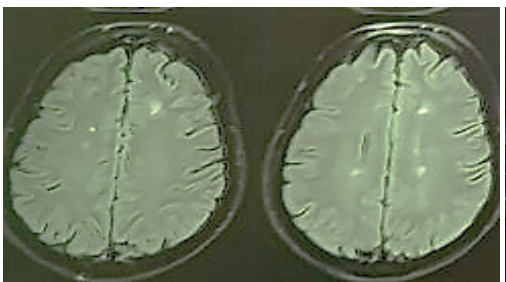

Case 1

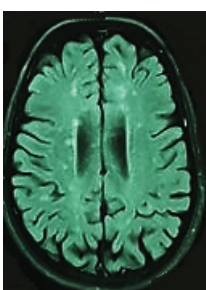

Case 2

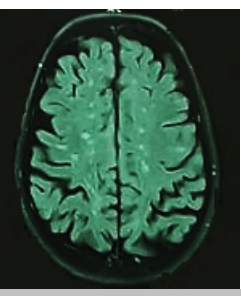

Case 3 with methylprednisolone and became symptomfree and remained asymptomatic during one year. In October 2018, she presented a blurred vision, ocular redness and also a pain in her left eye. Sclerouveitis was diagnosed for her by an ophthalmologist and then topical and oral corticosteroid was begun which helped to become symptoms free after 2 weeks. The immunologic tests and the laboratory analysis were reported to be negative. Cerebrospinal fluid analysis showed positive OCB with eleven bands. The medication was started by Rituximab.

\section{Discussion and Conclusion}

It is claimed that the prevalence of uveitis in MS is significantly higher than in general population in which the incidence of uveitis has been estimated between 17 and 52 per 100000 of population per year, and the prevalence as 38714 cases per 100000 of the population [16].

Currently, it is widely accepted that MS is likely a multiple disorder which contains several disease processes having similar presentations. For instance, uveitis which can be diagnosed in MS patients is assumed as secondary to an inflammatory event in the CNS which causes the immune system to become sensitized to antigens which are co-expressed in both uvea and CNS $[17,18]$. Besides, in an animal study, it was shown that myelin basic protein and myelin oligodendrocyte glycoprotein, the two involved antigens in MS, could simultaneously induce uveitis [19].

In the current study, the three cases of MS and uveitis are reported in detail. The diagnosis of MS was confirmed by neurologists based on Mc Donald's criteria and also uveitis was diagnosed by an ophthalmologist using SUN-criteria. It is reported that uveitis can be provoked in three different modes in patients; before, simultaneously but mostly after the onset of MS $[6,20]$. In the present investigation, uveitis has developed after the onset of MS in all of the patients.

In an epidemiologic study on uveitis which was designed by Soheilian et al [21] in Iran, sarcoidosis (7.3\%) and MS (4.2\%) were regarded as two most common systemic diseases, which were related to intermediate uveitis. In most of the articles, intermediate uveitis reported being the most frequent type of uveitis in comparison with other types [22]. In the current study, among the three patients, two showed intermediate and one had anterior uveitis.

Uveitis can be presented by a blurred vision which is also a common symptom of optic neuritis. Since, it is far important to differentiate the two ocular manifestations, blurred vision, and painful eye movements, which are always presented in optic neuritis help to distinguish uveitis from optic neuritis. After ruling out optic neuritis with common clinical examinations, like checking Marcus Gunn pupil [23], the patient is recommended to be visited by an ophthalmologist for diagnosing uveitis which can be a serious threatening factor for vision. After that, the patient should be referred back to a neurologist to follow up the disease management. It is notable that uveitis is mostly idiopathic; however, it could be due to some other disorders, such as sarcoidosis, rheumatologic diseases, autoimmune diseases and also infectious disease, like Lyme disease, tuberculosis, toxoplasmosis and syphilis [21]. In the current study, it is suggested that a neurologist should rule out other common causes of uveitis using Chest X-Ray (CXR), tuberculin test, Human Leukocyte Antigen (HLA-B27) and also rheumatologic evaluations. After confirmation uveitis by such evaluations, it can be considered to be idiopathic or relation to MS. Patients with MS usually suffer from uveitis ten times higher than normal population [24]. A study which was conducted by Ewald Lindner [25] and his 
colleague suggested the association of rs2104286 polymorphism with intermediate uveitis, but not with HLA-B27-associated acute anterior uveitis. Also, another evidence suggested that the immunopathological processes in MS and MSassociated uveitis could be quite similar, which means that MS and intermediate uveitis could share common immunogenetic predisposition (HLA-DR15) [26]. In addition, it is also shown that $T$ lymphocytes from MS patients react similarly to myelin basic protein and uveitogenic non-myelin protein arrestin [27]. As a matter of fact, there could be significant amino acid homology between immunodominant peptide sequences of the two studied proteins [27]. Also animal study showed evidence regarding that non-myelin protein, which was shared by the retina, uvea, and brain, might be involved in the pathogenesis of MS. Indeed, autoimmune encephalomyelitis, systemic administration of $\mathrm{T}$ cells specific for astrocyte-derived S100B protein co-expressed in the Muller cells of the retina resulted in an extensive inflammation of cerebral white and gray matter along with panuveitis and retinitis [28] Malinowski et al [29] and Biousse et al [30], noted that $11.1 \%$ and $20 \%$ of their patients with pars planitis had a family history of MS, respectively and hence they concluded that there might be a similar genetic predisposition of the development of MS and uveitis suggesting that such particular type of uveitis might be linked specifically to development of MS. Those results were in parallel with a letter which was provided by Farzin Forooghian [31] and suggested that the inclusion of MS-associated uveitis in diagnostic criteria of MS, as well as recognition of uveitis as an MS attack, could potentially lead to earlier diagnosis and treatment of MS and also MS attacks. Meanwhile, a pooled analysis which was expressed by Lyndell L. Lim [32] showed that the patients with uveitis showed a significantly shorter time in the first relapse (mean, 2.11 vs 8.12 years; $P=0.047$ ) and a dramatic higher ARR (0.31 vs $0.21 ; P=0.025)$ than those without uveitis. Due to the importance of disease management, a medication with the lowest adverse effect coupled with the highest beneficial impact on both uveitis and MS should be chosen for patients. For example, Becker et al [33] reported that MS-associated uveitis showed a good response to Interferons (IFNs). Also, based on a study which was conducted by Velazquez-
Villoria [34] IFN $\beta$ or glatiramer acetate could be effective in reducing uveitis episodes in the patients with MS-associated uveitis which was well tolerated in the majority of patients. Alireza Hedayatfar [35] in a retrospective, nonrandomized interventional case series study reported that mycophenolate mofetil showed beneficial effects on vision and intraocular inflammation with an acceptable safety profile. While Léa Jouve et al [36] confirmed that MSrelated uveitis has often a favorable evolution. It was shown that the patients who were on IFN $\beta$ had more severe and chronic uveitis; therefore, the authors were concerned that IFN $\beta$ given on the sole indication of uveitis could not be recommended. It was noted that if the steroidsparing agent could be needed for intraocular inflammation, immunosuppressive drugs should be considered.

As it was reported in some studies that MS was diagnosed after a few years of uveitis onset, hence, it is recommended to follow-up patients with unclear etiology of intermediate uveitis or vasculitis. FIGURE 2 depicts the summary of the current study and its conclusion.

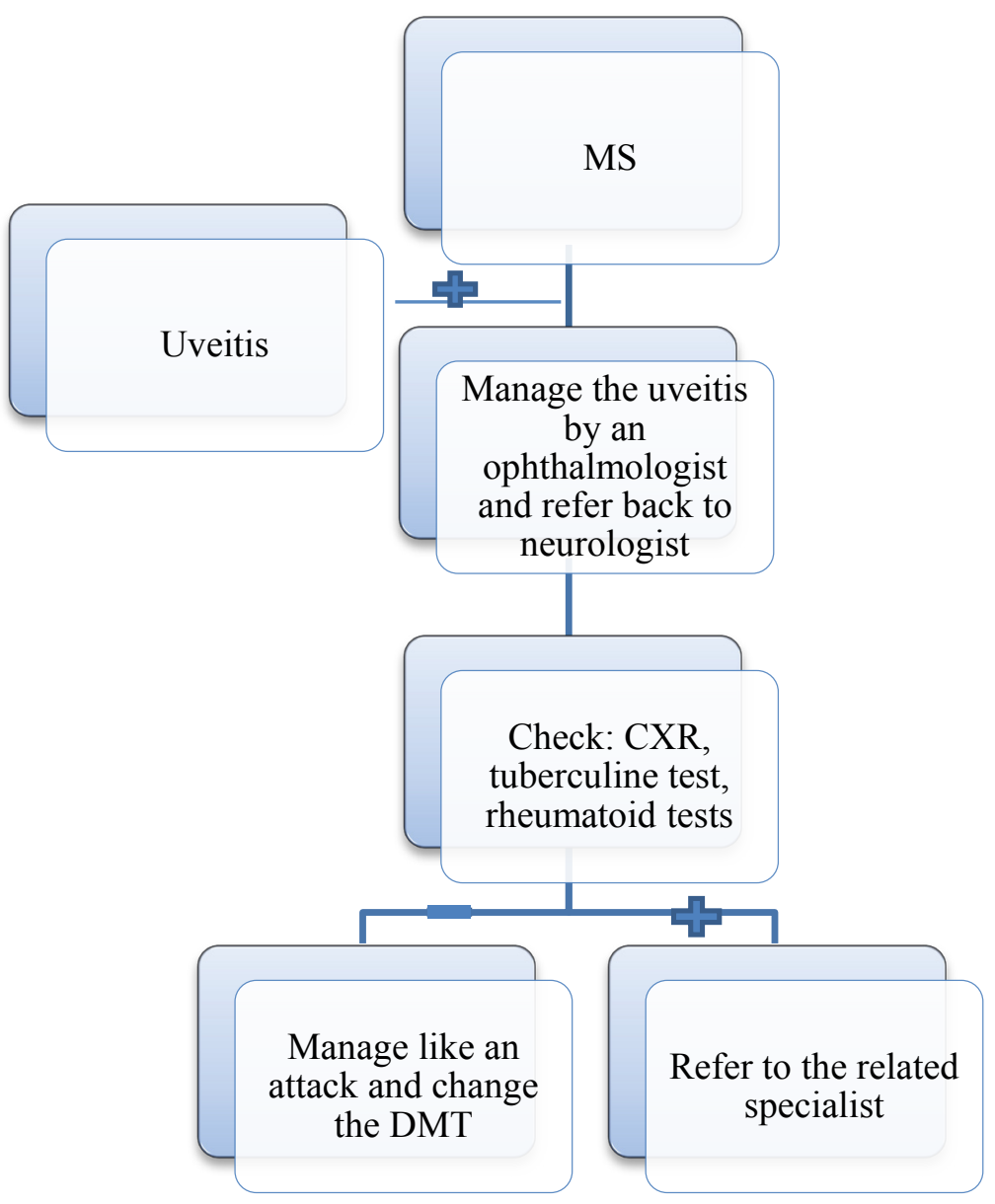

FIGURE 2: Approach of uveitis in MS patients. 


\section{Declarations}

Ethics approval and consent to participate

Written informed consent was obtained from every participant.

\section{Consent for Publication}

Written informed consent was obtained from the patient for publication of this Case report and any accompanying images. A copy of the written consent is available for review by the Editor of this journal.

\section{Availability of data and materials}

All data generated or analyzed during this study can be obtained from the corresponding author on reasonable request.

\section{Competing Interests}

The authors declare that they have no competing interests.

\section{Authors' Contributions}

SKR contributed to this work by recruiting the participants, obtaining the clinical information and drafting the manuscript. SS supervised the data analysis and revised the manuscript. SN, MHN, HP, SK, and AA contributed to revising the manuscript. All authors have read and approved the manuscript and ensure that this is the case.

\section{Funding}

No funding was obtained for this study.

\section{Acknowledgments}

We would like to thank the patient and his family for their participation in this study. 


\section{References}

Chen L, Gordon LK. Ocular manifestations of multiple sclerosis. Curr. Opin. Ophthalmol. 16(5), 315-320 (2005).

Windmüller M. Über die Augenstörungen bei beginnender multipler Sklerose. Deutsch Z. Nervenheilk. 39(1-2), 1-35 (1910).

Archambeau P, Hollenhorst R, Rucker CW. Posterior uveitis as a manifestation of multiple sclerosis. Mayo Clin. Proc. 40, 544-551 (1965).

Breger BC, Leopold IH. The incidence of uveitis in multiple sclerosis. Am. J. Ophthalmol. 62(3), 540-545 (1966).

Tola M, Granieri E, Casetta I, et al. Retinal periphlebitis in multiple sclerosis: a marker of disease activity? Eur. Neurol. 33(2), 93-96 (1993).

Shugaiv E, Tüzün E, Kürtüncü M, et al. Uveitis as a prognostic factor in multiple sclerosis. Mult. Scler. 21(1), 105107 (2015).

Olsen TG, Frederiksen J. The association between multiple sclerosis and uveitis. Surv. Ophthalmol. 62(1), 89-95 (2017).

Ardouin M, Urvoy M, Clement J, et al. Uveitis and multiple sclerosis: myth or reality? J. Fr. Ophtalmol. 2(2), 127-130 (1979).

Hogan MJ, Kimura SJ, Thygeson P. Signs and symptoms of uveitis*: I. Anterior uveitis. Am. J. Ophthalmol. 47(5), 155-170 (1959).

Group SoUNW. Standardization of uveitis nomenclature for reporting clinical data. Results of the First International Workshop. Am. J. Ophthalmol. 140(3), 509-516 (2005).

Zierhut M, Deuter C, Murray PI. Classification of uveitis-current guidelines. Eur. ophthalmic Review. 1, 77-78 (2007).

Gery I, Streilein JW. Autoimmunity in the eye and its regulation. Curr. Opin. Immunol. 6(6), 938-945 (1994).

Berger $\mathrm{T}$, Weerth S, Kojima K, et al. Experimental autoimmune encephalomyelitis: the antigen specificity of $\mathrm{T}$ lymphocytes determines the topography of lesions in the central and peripheral nervous system. Lab. Invest. 76(3), 355-364 (1997).

Schmidt S, Linington C, Zipp F, et al. Multiple sclerosis: comparison of the human T-cell response to $\mathrm{S} 100$ beta and myelin basic protein reveals parallels to rat experimental autoimmune panencephalitis. Brain. 120(8), 1437 1445 (1997).

McDonald WI, Compston A, Edan $\mathrm{G}$, et al. Recommended diagnostic criteria for multiple sclerosis: guidelines from the International Panel on the diagnosis of multiple sclerosis. Ann. Neurol. 50(1), 121-127 (2001).

Wakefield D, ChangJH. Epidemiology of uveitis. Int. Ophthalmol. Clin. 45(2), 1-13 (2005).

Shao H, Sun SL, Kaplan HJ, et al. Induction of autoimmune encephalomyelitis and uveitis in B6 and (B6x SJL) mice by peptides derived from myelin/oligodendrocyte glycoprotein. J. Neuroimmunol. 132(1-2), 117-122 (2002).

Manczak M, Jiang S, Orzechowska $\mathrm{B}$, et al. Crucial role of CCL3/MIP-1 $\alpha$ in the recurrence of autoimmune anterior uveitis induced with myelin basic protein in Lewis rats. J. Autoimmun. 18(4), 259270 (2002).

Messenger W, Hildebrandt L, Mackensen F, et al. Characterisation of uveitis in association with multiple sclerosis. Br. J. Ophthalmol. 99(2), 205209 (2015).

Schmidt S, Wessels L, Augustin A, et al. Patients with multiple sclerosis and concomitant uveitis/periphlebitis retinae are not distinct from those without intraocular inflammation. J. Neurol. Sci. 187(1-2), 49-53 (2001).

Soheilian M, Heidari K, Yazdani S, et al. Patterns of uveitis in a tertiary eye care center in Iran. Ocul. Immunol. Inflamm. 12(4), 297-310 (2004).

Zein G, Berta A, Foster S. Multiple sclerosis-associated uveitis. Ocul. Immunol. Inflamm. 12(2), 137-142 (2004).
O'Connor PS, Kasdon D, Tredici TJ, et al. The Marcus Gunn pupil in experimental tract lesions. Ophthalmology. 89(2), 160-164 (1982).

Hildebrandt A, Mackensen F. Uveitis bei multipler Sklerose: Übersicht und Ausblick (Leitthema). Der. Ophthalmologe. 111(8), 733-739 (2014).

Lindner E, Weger M, Steinwender $\mathrm{G}$, et al. IL2RA gene polymorphism rs2104286 $A>G$ seen in multiple sclerosis is associated with intermediate uveitis: possible parallel pathways? Invest. Ophthalmol. Vis. Sci. 52(11), 8295-8299 (2011).

Raja SC, Jabs DA, Dunn J, et al. Pars planitis: clinical features and class II HLA associations. Ophthalmology. 106(3), 594599 (1999).

Forooghian F, Cheung RK, Smith WC, et al. Enolase and arrestin are novel nonmyelin autoantigens in multiple sclerosis. J. Clin. Immunol. 27(4), 388396 (2007).

Kojima K, Berger T, Lassmann $\mathrm{H}$, et al. Experimental autoimmune panencephalitis and uveoretinitis transferred to the Lewis rat by $\mathrm{T}$ lymphocytes specific for the $S 100$ beta molecule, a calcium binding protein of astroglia. J. Exp. Med. 180(3), 817-829 (1994).

Malinowski SM, Pulido JS, Folk JC. Long-term visual outcome and complications associated with pars planitis. Ophthalmology. 100(6), 818-825 (1993).

Biousse V, Trichet C, Bloch ME, et al. Multiple sclerosis associated with uveitis in two large clinic-based series. Neurology. 52(1), 179-181 (1999).

Forooghian F. Uveitis and the diagnosis of multiple sclerosis. Can. J. Neurol. Sci. 44(3), 334-335 (2017).

Lim LL, Silva DG, Lo TC, et al. Uveitis in patients with multiple sclerosis in clinical trials of fingolimod: incidence, prevalence and impact on disease course. Ophthalmology. 6420(17), 33667-33669 (2018).

Becker M, Heiligenhaus A, Hudde T, 
et al. Interferon as a treatment for uveitis sclerosis. Arch. Soc. Esp. Oftalmol. 92(6), associated with multiple sclerosis. Br. J. 273-279 (2017).

Ophthalmol. 89(10), 1254-1257 (2005).

Velazquez VD, Macia BC, Segura GA, et al. Efficacy of immunomodulatory treatment with interferon- $\beta$ or glatiramer acetate in uveitis associated with multiple
Hedayatfar A, Falavarjani KG, Soheilian M, et al. Mycophenolate mofetil for the treatment of multiple sclerosisassociated uveitis. Ocul. Immunol. Inflamm. 25(3), 308-314 (2017).
Jouve L, Benrabah R, Héron E, et al. Multiple sclerosis-related uveitis: does MS treatment affect uveitis course? Ocul. Immunol. Inflamm. 25(3), 302-307 (2017). 